\title{
Evaluation of risk stratification and sepsis-related mortality in patients with positive blood cultures via procalcitonin, combined with the PIRO system in ED
}

\section{Long Yang}

Beijing Chao-Yang Hospital

Yue Lin

China-Japan Friendship Hospital

\section{Xiangqun Zhang}

Beijing Chao-Yang Hospital

\section{Bing Wei}

Beijing Chao-Yang Hospital

\section{Xinhua Ye}

Beijing Chao-Yang Hospital

Jun Yang

Beijing Chao-Yang Hospital

Jianmei Song

Beijing Chao-Yang Hospital

Junyu Wang

Beijing Chao-Yang Hospital

Bo Liu (D ripplecyyy@126.com )

China-Japan Friendship Hospital

\section{Research Article}

Keywords: Procalcitonin, PIRO score, positive blood culture, ICU, MODS, 28-day mortality

Posted Date: June 9th, 2022

DOI: https://doi.org/10.21203/rs.3.rs-1408466/v2

License: (c) (i) This work is licensed under a Creative Commons Attribution 4.0 International License.

Read Full License 


\section{Abstract}

Background: The aim of the study was to evaluate risk stratification and sepsis-related mortality in patients with positive blood cultures via procalcitonin (PCT) combined with the predisposition囚infection, response, and organ dysfunction (PIRO) system in emergency departments (ED).

Methods: A total of 1074 patients with positive blood cultures were admitted to Beijing Chao-Yang Hospital ED from December 2017 to October 2020. Their serum PCT was recorded, along with a Sequential Organ Failure Assessment (SOFA) score, Mortality in Emergency Department Sepsis (MEDS) score, Acute Physiology and Chronic Health Evaluation II (APACHE II) score, and PIRO score to predict the prognosis of septic patients with positive blood culture in terms of ICU (Intensive care unit) admission, multiple organ dysfunction syndrome (MODS) development, and 28-day mortality. Receiver operating characteristic (ROC) curves and logistic regression analysis were used to assess the prognostic value of the scoring systems.

Results: On admission, 978 patients met the inclusion criteria. PCT, MEDS, APACHE II, and PIRO scores were found to independently predict ICU admission, MODS development, and 28-day mortality $(P<0.05)$, whereas SOFA did not. The AUC values of the PCT, MEDS, APACHE II, and PIRO scores for ICU admission were $0.620,0.740,0.780$, and 0.751 , respectively. For MODS, the AUC values of PCT, MEDS, APACHE II, and PIRO were $0.664,0.701,0.761$, and 0.811 , respectively. In the prediction of 28-day mortality, the AUC values of PCT, MEDS, APACHE II, and PIRO were $0.782,0.745,0.805$, and 0.831 , respectively. The AUC values combined PCT and PIRO system in predicting MODS and 28-day mortality were better than when predicting ICU admission. Meanwhile, the AUC values of PCT when combined with MEDS, APACHE II, or PIRO score were higher than that of any single predictor.

Conclusions: This study indicates that PCT combined with the PIRO scoring system has a higher predictive value and is superior in predicting MODS and 28-day mortality in septic patients with a positive blood culture.

\section{Background}

Sepsis is a leading cause of high morbidity and mortality [1]. Severe sepsis and septic shock remain the dominant cause of death in the United States [2]. Mortality from severe sepsis and septic shock remains unacceptably high, ranging from 20 to $40 \%$, depending on the illness severity [3].

Approximately 200,000 patients are affected by bacteremia each year, with 10 cases per 1000 hospital admissions [4,5]. Bacteremia has high mortality, and due to the obvious dangers of undertreatment, many physicians liberally order cultures [6]. Blood cultures are generally considered to be the most sensitive method of detecting bacteremia and are useful in certain diagnoses and critically ill patients[7,8]. The blood culture results can be used to guide the adjustment of antibiotics regimes [9]. Positive blood culture usually indicates a serious infection with fever, chills, leukocytosis, focal infection, sepsis, or suspected endocarditis, and has poor outcome [6]. However, we found that a large percentage of positive blood 
culture patients were not admitted to the ICU, and did not develop MODS, instead, following timely, systematic, and effective treatment, they were successfully discharged. Therefore, finding suitable predictors for the prognosis of patients with positive blood culture is highly important.

Consequently, our study evaluated the predictive performance of PCT combined with PIRO, in ICU admission, MODS development, and 28-day mortality in septic patients with positive blood cultures in the ED.

\section{Methods}

\section{Patients}

The study was conducted in Beijing Chao-Yang Hospital ED, a tertiary teaching hospital of Capital Medical University, and was approved by the human research ethics committee of Beijing Chao-Yang Hospital. A total of 1074 septic patients with positive blood cultures were enrolled in our cohort from 2017 to 2020, and written informed consent upon ED admission was acquired.

\section{Inclusion and exclusion criteria}

The inclusion criteria were as follows: (1) Patients signed informed consent forms; (2) Age >18 years; (3) Patients with sepsis according to Sepsis-3 (suspected or definite infection with an increase in SOFA score 22) [10]. (4) Positive blood culture results.

The exclusion criteria were as follows: (1) Age < 18 years; (2) Terminal stage of disease (malignant cancer with metastases, AIDS, end-stage renal or hepatic disease, chronic heart failure); (3) Viral or fungal infection. (4) Refusal to participate in the study by patients or their relatives or transferred to other hospitals.

\section{Data collection}

Patient characteristics were recorded including age, gender, vital signs, and past medical history. Whole blood leukocyte counts, blood gas indexes, and blood biochemistry were identified within 24h. The SOFA, MEDS, APACHE II, and PIRO scores were calculated according to vital signs, and laboratory test results. The Howell-PIRO scoring system [11] was applied in this study, and the criteria are shown in Table 1.

\section{Outcome variables}

MODS was defined as the fulfillment of two or more of the severe sepsis criteria at any time within 3 days of enrollment, excluding organ dysfunction, induced by pre-existing disease [12]. Organ function was assessed at enrollment and reassessed when deterioration occurred. All patients were followed up for 28 days through medical records or by telephone. ICU admission during follow-up, development of MODS within 3 days, and 28-day mortality were considered as the outcome criteria.

\section{Statistical analysis}


All data were analyzed by SPSS 25.0 (SPSS Inc., Chicago, IL, USA). Normally distributed data, expressed as the mean \pm standard deviation, was compared using an independent-samples $t$ test. Data with skewed distribution was expressed as the median and quartiles, and was analyzed via a Mann-Whitney U test, and a chi-square test was used to compare frequencies. Logistic regression analysis was used to determine the independent outcome predictors. Receiver operating characteristic (ROC) curves were constructed and the area under the curves (AUC) was determined to assess predictive value. The survival curve was estimated by Prism 6.0. All statistical tests were two-tailed, and $P<0.05$ was considered statistically significant.

\section{Results}

\section{Characteristics of the study cohort}

A total of 1074 septic patients with positive blood cultures in the ED between 2017 and 2020 were enrolled in this study. 71 patients had a viral or fungal infection, 10 patients refused to participate, and 15 patients were transferred to other hospitals. Finally, 978 participants completed the 28 follow-up days (Fig.1).

In our study, 978 septic patients with positive blood cultures were divided into ICU ( $n=284)$ and non-ICU $(n=694)$ admission groups. When comparing the characteristics of these patients, we found that there were significant differences in age $(P=0.040)$, chronic obstructive pulmonary disease (COPD) $(P=0.000)$, hypertension ( $P=0.001)$, chronic heart failure (CHF) $(P=0.007)$, mean arterial pressure (MAP) $(P=0.000)$, heart rate (HR) $(P=0.000)$, white blood cells (WBC) $(P=0.000)$, lactate (Lac) $(P=0.000)$, PCT $(P=0.000), C$ reactive protein (CRP) $(P=0.000)$, albumin (ALB) $(P=0.000)$, SOFA $(P=0.000)$, MEDS $(P=0.000)$, APACHE II $(P=0.000)$, and PIRO $(P=0.000)$ between ICU and non-ICU admission groups (Table 2, Fig. 1$)$.

In addition, we also divided the participants into two other categories, MODS $(n=204)$ and non-MODS $(\mathrm{n}=774)$ according to the development of MODS within 3 days. There were obvious differences in age $(P=0.001), \operatorname{MAP}(P=0.000), \operatorname{HR}(P=0.000)$, respiratory rate (RR) $(P=0.000), \mathrm{WBC}(P=0.000), \operatorname{Lac}(P=0.040)$, $\operatorname{PCT}(P=0.000), \operatorname{CRP}(P=0.000), \operatorname{ALB}(P=0.000)$, (NLR) $(P=0.000)$, SOFA $(P=0.000)$, MEDS $(P=0.000)$, APACHE II $(P=0.000)$, and PIRO $(P=0.000)$ between these two groups (Table2, Fig.1).

After 28 days follow-up, statistical differences were evident in age ( $P=0.001)$, chronic heart failure (CHF) $(P=0.001), \operatorname{MAP}(P=0.000), \operatorname{HR}(P=0.000), \mathrm{RR}(P=0.000), \mathrm{WBC}(P=0.000), \operatorname{Lac}(P=0.040), \mathrm{PCT}(P=0.000)$, $\operatorname{CRP}(P=0.000)$, ALB $(P=0.000)$, (NLR) $(P=0.000)$, SOFA $(P=0.000)$, MEDS $(P=0.000)$, APACHE $\|(P=0.000)$, and PIRO $(P=0.000)$ between survivor $(n=797)$ and non-survivor $(n=181)$ groups (Table2, Fig. 1$)$.

The median of the SOFA, MEDS, and PIRO scores, and mean of the APACHE II score in ICU-admission, MODS development, and 28-day mortality groups are shown in Table2, Fig.2. The average SOFA, MEDS, APACHE II, and PIRO scores differed significantly between patients who did, and did not, meet the outcome criteria $(P=0.000)$. 


\section{Spearman's correlations between 28-day mortality and SOFA, MEDS, APACHE II or PIRO score.}

To investigate the correlations between 28-day mortality and SOFA, MEDS, APACHE II or PIRO score, Spearman's correlation analysis was performed. Significant negative liner correlations of 28-day mortality with SOFA, MEDS, APACHE II or PIRO score were presented in Fig.3A-D ( $r$ values were $-0.62,-0.75,-0.64$, -0.49 respectively, and all $P<0.001)$.

\section{Binary logistic regression analysis of the prognostic outcome of septic patients with positive blood cultures in the ED}

Binary logistic regression was used to analyze the independent predictors in our study cohort. The results showed that WBC $(\beta=0.176$, odds ratio $(\mathrm{OR})(95 \% \mathrm{Cl})=1.192(1.031-1.379), P=0.018), \mathrm{PCT}(\beta=0.241, \mathrm{OR}$ $(95 \% \mathrm{Cl})=1.273(1.037-1.563), P=0.021), \operatorname{Lac}(\beta=1.261, \mathrm{OR}(95 \% \mathrm{Cl})=3.530(1.867-6.676), P=0.000)$, ALB $(\beta=-0.142$, OR $(95 \% \mathrm{Cl})=0.867$ (0.792-0.949), $P=0.002), \operatorname{NLR}(\beta=0.077, \mathrm{OR}(95 \% \mathrm{Cl})=1.080(1.011-1.155)$, $P=0.023)$, MEDS score $(\beta=0.895$, OR $(95 \% \mathrm{Cl})=2.447$ (1.607-3.728), $P=0.000)$, APACHE II score $(\beta=0.623$, $\mathrm{OR}(95 \% \mathrm{Cl})=1.864(1.405-2.474), P=0.000)$ and $\mathrm{PIRO}$ score $(\beta=0.328, \mathrm{OR}(95 \% \mathrm{Cl})=1.388(1.118-1.723)$, $P=0.003)$ were the independent predictors of ICU admission, but the SOFA score $(\beta=0.220$, OR $(95 \% \mathrm{Cl})$ $=2.701$ (1.548-4.713), $P=0.120$ ) was not (Table 3).

In MODS development, WBC $(\beta=0.362$, OR $(95 \% \mathrm{Cl})=1.436$ (1.115-1.849), $P=0.005), \mathrm{PCT}(\beta=0.136$, OR $(95 \% \mathrm{Cl})=1.145(0.953-1.377), P=0.048)$, ALB $(\beta=-0.665$, OR $(95 \% \mathrm{Cl})=0.514(0.353-0.750), P=0.001), \mathrm{NLR}$ $(\beta=0.270$, OR $(95 \% \mathrm{Cl})=1.310(1.092-1.572), P=0.004)$, MEDS score $(\beta=0.383$, OR $(95 \% \mathrm{Cl})=1,466(1.074-$ $2.001), P=0.016)$, APACHE II score $(\beta=0.372$, OR $(95 \% \mathrm{Cl})=1.450(1.056-1.990), P=0.022)$, and PIRO score $(\beta=0.196, \mathrm{OR}(95 \% \mathrm{Cl})=1.216(1.032-1.434), P=0.019)$ were the independent predictors of developing MODS, but the SOFA scores $(\beta=0.001$, OR $(95 \% \mathrm{Cl})=1.001(0.660-1.518), P=0.995)$ and Lac $(\beta=0.177$, OR $(95 \% \mathrm{Cl})=1.194(0.664-2.145), P=0.554)$ determined it was not a predictor (Table 3$)$.

In addition, with 28-day mortality, the results showed that WBC $(\beta=0.996, \mathrm{OR}(95 \% \mathrm{Cl})=2.707(1.353-$ 5.417), $P=0.005)$, PCT $(\beta=0.306$, OR $(95 \% \mathrm{Cl})=1.358$ (1.121-1.644), $P=0.002)$, Lac $(\beta=1.822$, OR $(95 \% \mathrm{Cl})$ $=6.184$ (1.565-24.436), $P=0.009)$, MEDS score $(\beta=0.440$, OR $(95 \% \mathrm{Cl})=1.553(1.163-2.074), P=0.003)$, APACHE II score $(\beta=0.484$, OR $(95 \% \mathrm{Cl})=1.623(1.179-2.234), P=0.003)$, and PIRO score $(\beta=0.322$, OR $(95 \% \mathrm{Cl})=1.380(1.085-1.754), P=0.009)$ were the independent predictors of 28-day mortality, but SOFA $(\beta=0.092$, OR $(95 \% \mathrm{Cl})=1.096(0.805-1.492), P=0.560), \mathrm{NLR}(\beta=0.128, \mathrm{OR}(95 \% \mathrm{Cl})=1.136(0.843-1.533)$, $P=0.402)$, and ALB $(\beta=-0.226$, OR $(95 \% \mathrm{Cl})=0.798(0.636-1.001), P=0.051)$ were not (Table 3).

\section{Prediction of the prognostic outcomes of septic patients with positive blood cultures}

The ROC curves of the PCT, combined with the severe scores for predicting outcomes in septic patients with positive blood cultures, for ICU-admission, MODS development, and 28-day mortality are shown in Fig.4, and the AUCs are presented in Tables 4,5,and 6. The AUC values of the PCT, MEDS, APACHE II, and PIRO scores for ICU-admission were 0.620 (95\% Cl: 0.533-0.706, $P=0.009$ ), 0.740 (95\% Cl: 0.663-0.817, $P=0.000), 0.780$ (95\% Cl: $0.709-0.850, P=0.000), 0.751(95 \% \mathrm{Cl}: 0.674-0.828, P=0.000)$, respectively. The 
AUC values of a combination of PCT and severe scores were as follows: PCT+MEDS: 0.772 (95\% Cl: 0.698-0.846, $P=0.000$ ); PCT+APACHE II: 0.821 (95\% Cl: 0.757-0.885, $P=0.000$ ); PCT+PIRO: 0.795 (95\% Cl: 0.723-0.867, $P=0.000$ ). The predictive ability of APACHE II (AUC:0.780) for ICU admission was slightly better than that of MEDS (AUC: 0.740) and PIRO (AUC:0.751). Consequently, PCT+APACHE II (AUC:0.821) is much better at predicting ICU admission than PCT+MEDS (AUC:0.772) and PCT+PIRO (AUC:0.795) (Fig.4A and Table 4).

Furthermore, for MODS incidence, the AUC values of the PCT, MEDS, APACHE II, and PIRO scores were 0.664 (95\% Cl: 0.584-0.745, $P=0.000$ ), 0.701 (95\% Cl: 0.622-0.779, $P=0.000), 0.761$ (95\% Cl: 0.691-0.832, $P=0.000), 0.811$ (95\% Cl: 0.746-0.876, $P=0.000$ ), respectively. The AUC values of the combination of PCT and severity scores were as follows: PCT+MEDS: 0.758 (95\% Cl: 0.685-0.831, $P=0.000)$; PCT+APACHE II: 0.794 (95\% Cl: $0.727-0.862, P=0.000$ ); PCT+PIRO: 0.837 (95\% Cl: $0.776-0.898, P=0.000$ ). The ability of PIRO (AUC:0.811) to predict the onset of MODS was much better than that of MEDS (AUC: 0.701 ) and APACHE II (AUC: 0.761). Therefore, PCT+PIRO (AUC: 0.837) would be better used in predicting the development of MODS than PCT+MEDS (AUC: 0.758) and PCT+APACHE II (AUC: 0.794) (Fig.4B and Table $5)$.

In addition, after 28-days follow-up, the AUC values of the PCT, MEDS, APACHE II, and PIRO scores for 28day mortality were 0.782 (95\% Cl: 0.683-0.882, $P=0.000$ ), 0.745 (95\% Cl: 0.627-0.863, $P=0.000$ ), 0.805 (95\% Cl: $0.707-0.904, P=0.000), 0.831$ (95\% Cl: $0.742-0.920, P=0.000)$, respectively. The AUC values of the combination of PCT and severity scores were as follows: PCT+MEDS: 0.812 (95\% Cl: 0.705-0.919, $P=0.000$ ); PCT+APACHE II: 0.866 (95\% Cl: 0.789-0.943, $P=0.000$ ); PCT+PIRO: 0.885 (95\% Cl: 0.810-0.961, $P=0.000$ ). The ability of the PIRO score (AUC: 0.831 ) to predict 28-day mortality was better than that of MEDS (AUC: 0.745 ) and APACHE II (AUC: 0.805). Thus, PCT+PIRO (AUC: 0.885) are slightly better at predicting 28-day mortality than PCT+MEDS (AUC: 0.812) and PCT+APACHE II (AUC: 0.866) (Fig.4C and Table 6). Furthermore, the survival curve was estimated by Prism 6.0. The results show that 28-day mortality was around $18.4 \%$, which is an improvement on the $27.9 \%$ measured 5 years ago in this hospital (Fig.5).

\section{Discussion}

Bloodstream infections (BSIs) are commonly considered as being caused by the presence of viable microorganisms in blood [13], and are often associated with severe infectious diseases with excessively high morbidity and mortality rates [14-16]. Blood cultures are considered to be the reference standard for diagnosing BSIs and are still indispensable in BSI diagnosis [13]. The Surviving Sepsis Campaign guidelines recommend that blood cultures are taken before starting antimicrobial therapy [17]. Blood cultures are straightforward to perform and exhibit high sensitivity to cultivable pathogens [18]. The worldwide increases in incidence have emerged as one of the leading causes of death [19], particularly in critically ill patients [20], and the global burden of morbidity and mortality from sepsis is significant [21]. However, improvements in the clinical outcomes of patients with sepsis and septic shock have been made within the last decade, when short-term mortality rates had routinely approached $20 \%$ [22]. Delayed, 
insufficient, or inappropriate anti-infection treatment leads to increased mortality from BSIs $[14,16,23]$. Prompt initiation of infective antimicrobial therapies is a critical survival determinant [24]. Currently, the cornerstone of BSI sepsis management continues to be early antimicrobial administration, source control, and supportive care [25-28]. Therefore, early recognition of sepsis criticality, and immediate intervention is significant.

However, there have been very few studies on the criticality and risk structure of septic patients with BSIs. We did find that not all patients with positive blood cultures have a poor prognosis. After timely and effective treatment, many of them were successfully discharged. Therefore, it is necessary to find suitable predictors for the outcomes and prognosis of positive blood culture sepsis. We used SOFA, MEDS, APACHE II, and PIRO scoring systems to study the risk stratification and prognosis of septic patients. The concept of the predisposition, infection, response, and organ dysfunction (PIRO) scoring system was presented at the 2001 International Sepsis Definitions Conference, to improve on traditional sepsis classification [29]. The PIRO system is an ideal staging system that incorporates assessment of premorbid baseline susceptibility, specific disorders responsible for illness, host response to infection, and the resulting degree of organ dysfunction. The four components of the PIRO system cover multiple independent factors that may influence the onset, development, and outcome of sepsis [12]. Since the first PIRO system appeared in 2008, many different PIRO systems have been developed. The PIRO system mainly used to study ICU populations with ICU specific features is not suitable for the ED. A new PIRO system developed by Howell is designed for bedside use and is superior to other PIRO systems [30]. Our study applied the Howell-PIRO scoring system to predict the risk stratification and prognosis of septic patients with positive BSIs in an ED. Previous studies showed that SOFA, MEDS, and APACHE II scores increased with sepsis progression, reaching a maximum limit in septic shock. Therefore, the above four scoring systems were used to assess the prognosis of sepsis with positive BSIs.

In addition to the critical sepsis scoring system, several of the most common clinical biomarkers are used to assess the prognosis of patients with positive bloodstream infections. PCT, a prohormone of calcitonin, encoded by the calcitonin-I(CALC-1) gene on chromosome 11, has successfully detected infection, as well as providing antibiotic management guidance [31]. It has been used extensively as a biomarker in sepsis diagnosis, risk stratification, prognosis evaluation, and therapy monitoring in recent years. Early lactate levels are associated with organ dysfunction and mortality in the ICU and ED. Sepsis-3 with a recommended serum lactate level $>2 \mathrm{mmol} / \mathrm{L}$ is a major criterion in the clinical identification of septic shock [10]. A lactate concentration greater than $4 \mathrm{mmol} / \mathrm{L}$ is described as having $96 \%$ specificity in predicting hospital mortality in non-hypotensive patients [32]. NLR, as a new risk factor in the diagnosis of sepsis, has attracted substantial attention. Sepsis elevates neutrophil levels and decreases lymphocyte counts resulting from pathogenic microorganisms infection, and therefore has a higher NLR level [33]. Higher NLR levels are therefore a helpful predictor of sepsis and patients with a higher likelihood of an unfavorable prognosis [34].

In our study, SOFA, MEDS, APACHE II, and PIRO scores, as well as PCT, Lac, and NLR were used to predict the prognosis of sepsis in positive BSI patients. As for ICU admission, binary logistic regression analysis 
showed that WBC, PCT, Lac, ALB, NLR, along with the MEDS, APACHE II, and PIRO scores were independent predictors, but the SOFA score was not. The AUC of the APACHE II score was 0.780 , which was a little higher than that of PCT (0.620), MEDS (0.740), or PIRO (0.751). In addition, the AUC of PCT in combination with the APACHE II score was significantly higher than that of PCT+MEDS. The AUC of PCT in combination with the APACHE II score was slightly higher than that of PCT+PIRO. The APACHE II score is regularly used to evaluate sepsis severity. For patients admitted to the ICU, their condition is usually critical and they are therefore more likely to develop sepsis or sepsis shock. Our results indicate that the APACHE II score has the highest predictive value for septic patients with BSIs. Previous studies have shown that the PIRO scoring system is suitable in assessing ICU patient criticality [35]. This is consistent with our results. The AUC of the PIRO score was comparable with the APACHE II score, and similarly had a high predictive value of ICU admission. No single biomarker predictions were particularly high, but when combined with other predictors the prediction value increases significantly.

MODS often indicates disease aggravation and deterioration, and is often associated with short-term mortality [36]. In our study, WBC, PCT, NLR, ALB, and the MEDS, APACHE II, and PIRO scores independently predicted MODS development within three days of ED arrival. However, those of Lac and SOFA did not. The AUC of the PIRO score (0.811) was higher than that of the PCT (0.664), MEDS (0.701), or APACHE II (0.761) scores. Meanwhile, the AUC of PCT combined with the PIRO score was significantly higher than that of any single predictor. The AUC of PCT in combination with the PIRO score was slightly higher than that of PCT+MEDS or PCT+APACHE II score. The PIRO scoring system incorporates the highrisk factors of MODS and variable reflected established organ dysfunction [12]. Therefore, its predictive value was found to be better than both the APACHE II and MEDS scores. These results correspond with the conclusions of previous studies.

In addition, we analyzed the 28-day mortality of positive BSIs. PIRO had a higher predictive value and showed better predictive ability than MEDS or APACHE II scores. In terms of combined prediction, PCT combined with the PIRO score had the highest predictive value for septic patients with positive BSIs. This was consistent with the characteristics of the PIRO scoring system used to assess the severity of critically ill patients. For 28-day mortality, the PIRO scoring system was superior and therefore could be widely used in sepsis prognosis assessment.

Our study had several limitations: It was limited by being a single-center study with a relatively small sample size, and more centers and samples needed to be added to the cohort; some biomarkers had limitations and could not represent all the characteristics of sepsis, and more evaluation biomarkers were needed in the study. Our study also had some positives, in that this was the first time, to our knowledge, that a combination of the PIRO scoring system and PCT has successfully been used to assess the criticality and prognosis of septic patients with positive BSIs.

\section{Conclusion}


This study indicates that PCT combined with the PIRO scoring system is superior to that of PCT+MEDS or PCT+APACHE II score in predicting MODS and 28-day mortality in patients with positive blood cultures. The higher predictive value of the PIRO score is therefore essential in assessing the prognosis of sepsis patients with positive BSIs.

\section{Abbreviations}

ICU, Intensive care unit; MODS: multiple organ dysfunction syndrome; COPD, chronic obstructive pulmonary disease; CVD, cerebrovascular disease; $\mathrm{CHF}$, congestive heart failure; $\mathrm{CRD}$, chronic renal disease; DM, diabetes mellitus; MAP, mean arterial pressure; WBC, white blood cell; PCT, procalcitonin; Lac, lactate; CRP :C-reactive protein; ALB, albumin; NLR, neutrophils and lymphocytes ratio; SOFA, Sequential Organ Failure Assessment; APACHE II, Acute Physiology and Chronic Health Evaluation II; MEDS, Mortality in emergency department sepsis; PIRO, Predisposition, Infection, Response, and Organ dysfunction

\section{Declarations}

\section{Acknowledgements}

We would like to thank Beijing Chao-Yang Hospital for facilitating the study and covering the data collection costs. We would also like to acknowledge all data collectors, supervisors, and respondents without whom this research would not have been realized.

\section{Authors' contributions}

LY and YL contributed equally to this work and should be regarded as co-first authors. BL and JW have conceived the study and interpreted the results. LY drafted and revised the manuscript. YL have performed the statistical analyses and revised the manuscript. $\mathrm{XZ}, \mathrm{BW}, \mathrm{XY}$, JY and JS collected original data and revised the manuscript for important intellectual content. All authors have read and approved the submitted manuscript.

\section{Funding}

This research did not receive any specific grant from funding agencies in the public, commercial, or notfor-profit sectors.

\section{Availability of data}

All relevant data have been published in the paper.

\section{Declarations}

Ethical approval and consent to participant 
All procedures performed in studies involving human participant were in accordance with the ethical standards of the institutional and with the 1964 Helsinki declaration and its later amendments or comparable ethical standards. The study was approved by Beijing Chao-Yang Hospital Ethics Committee. Informed consent was obtained from all individual participants included in the study.

\section{Consent for publication}

Not applicable.

\section{Conflict of interest}

The authors declare that they have no conflict of interest.

\section{References}

1. Martin GS. Sepsis, severe sepsis and septic shock: changes in incidence, pathogens and outcomes. Expert Rev Anti Infect Ther 2012; 10(6):701-706 https://doi: 10.1586/eri.12.50.

2. Kethireddy S, Bilgili B, Sees A, Kirchner H, Ofoma U, Light R, et al. Culture-Negative Septic Shock Compared With Culture-Positive Septic Shock: A Retrospective Cohort Study. Crit Care Med 2018; 46: 506-512. https://DOI: 10.1097/CCM.0000000000002924

3. Deutschman CS, Tracey KJ. Sepsis: current dogma and new perspectives. Immunity 2014; 40: 463475. https://doi: 10.1016/j.immuni.2014.04.001.

4. Lee C-C, Chen S-Y, Chang I-J, Chen S-S, Wu S-C. Comparison of clinical manifestations and outcome of community-acquired bloodstream infections among the oldest old, elderly, and adult patients. Medicine 2007; 86: 138-144. https://doi: 10.1097/SHK.0b013e318067da56.

5. Weinstein MP, Towns ML, Quartey SM, Mirrett S, Reimer LG, Parmigiani G, et al. The clinical significance of positive blood cultures in the 1990s: a prospective comprehensive evaluation of the microbiology, epidemiology, and outcome of bacteremia and fungemia in adults. Clin Infect Dis 1997; 24: 584-602. https://doi: 10.1093/clind/24.4.584.

6. Long B, Koyfman A. Best Clinical Practice: Blood Culture Utility in the Emergency Department. J Emerg Med 2016; 51: 529-539. https://doi: 10.1016/j.jemermed.2016.07.003.

7. Coburn B, Morris AM, Tomlinson G, Detksy A. Does this adult patient with suspected bacteremia require blood cultures? JAMA 2012; 308: 502-511. https://doi: 10.1001/jama.2012.8262.

8. Weinstein MP, Reller LB, Murphy JR, Lichtenstein K. The clinical significance of positive blood cultures: a comprehensive analysis of 500 episodes of bacteremia and fungemia in adults. I. Laboratory and epidemiologic observations. Rev Infect Dis 1983; 5: 35-53. https://doi: 10.1093/clinids/5.1.35.

9. Berild D, Mohseni A, Diep LM, Jensenius M, Ringertz S. Adjustment of antibiotic treatment according to the results of blood cultures leads to decreased antibiotic use and costs. J Antimicrob Chemother 2006; 57: 326-330. https://doi: 10.1093/jac/dki463. Epub 2005 Dec 30. 
10. Singer M, Deutschman CS, Seymour CW, Hair MS, Annane D, Bauer M. The Third International Consensus Definitions for Sepsis and Septic Shock (Sepsis-3). JAMA 2016; 315: 801-810. https://doi: 10.1001/jama.2016.0287.

11. Tafelski S, Nachtigall I, Stengel S, Wernecke K, Spies C. Comparison of three models for sepsis patient discrimination according to PIRO: predisposition, infection, response and organ dysfunction. Minerva Anestesiol 2015; 81: 264-271.

12. Chen Y-X, Li C-S. Risk stratification and prognostic performance of the predisposition, infection, response, and organ dysfunction (PIRO) scoring system in septic patients in the emergency department: a cohort study. Crit Care 2014; 18: R74. https://doi: 10.1186/cc13832.

13. Peker N, Couto N, Sinha B, Rossen J. Diagnosis of bloodstream infections from positive blood cultures and directly from blood samples: recent developments in molecular approaches. Clin Microbiol Infect 2018; 24: 944-955. https://doi: 10.1016/j.cmi.2018.05.007.

14. Opota O, Croxatto A, Prod'hom G, Greub G. Blood culture-based diagnosis of bacteraemia: state of the art. Clin Microbiol Infect 2015; 21: 313-322. https://doi: 10.1016/j.cmi.2015.01.003.

15. Rutanga JP, Nyirahabimana T. Clinical Significance of Molecular Diagnostic Tools for Bacterial Bloodstream Infections: A Systematic Review. Interdiscip Perspect Infect Dis 2016; 2016: 6412085. https://doi: 10.1155/2016/6412085.

16. Loonen AJM, Wolffs PFG, Bruggeman CA, Brule AJ. Developments for improved diagnosis of bacterial bloodstream infections. Eur J Clin Microbiol Infect Dis 2014; 33: 1687-1702. https://doi: 10.1007/s10096-014-2153-4. Epub 2014 May 22.

17. Dellinger RP, Levy MM, Rhodes A, Annane D, Gerlach H, Opal S, et al. Surviving sepsis campaign: international guidelines for management of severe sepsis and septic shock: 2012. Crit Care Med 2013; 41: 580-637. https://doi: 10.1097/CCM.0b013e31827e83af.

18. Dubourg G, Raoult D. Emerging methodologies for pathogen identification in positive blood culture testing. Expert Rev Mol Diagn 2016; 16: 97-111. https://doi: 10.1586/14737159.2016.1112274.

19. Heron M. Deaths: Leading Causes for 2014. Natl Vital Stat Rep 2016; 65: 1-96.

20. GBD 2013 Mortality and Causes of Death Collaborators. Global, regional, and national age-sex specific all-cause and cause-specific mortality for 240 causes of death, 1990-2013: a systematic analysis for the Global Burden of Disease Study 2013. Lancet 2015; 385: 117-171. https://doi:10.1016/S0140-6736(14)61682-2.

21. Reinhart K, Daniels R, Kissoon N, Machado F, Schachter R, Finfer S. Recognizing Sepsis as a Global Health Priority - A WHO Resolution. N Engl J Med 2017; 377: 414-417. https://DOI: 10.1056/NEJMp1707170.

22. Kaukonen K-M, Bailey M, Suzuki S, Pilcher D, Bellomo R. Mortality related to severe sepsis and septic shock among critically ill patients in Australia and New Zealand, 2000-2012. JAMA 2014; 311: 13081316. https://doi: 10.1001/jama.2014.2637.

23. Bearman GML, Wenzel RP. Bacteremias: a leading cause of death. Arch Med Res 2005; 36: 646-659. https://doi: 10.1016/j.arcmed.2005.02.005. 
24. Kumar A, Zarychanski R, Light B, Parrillo J, Maki D, Simon, et al. Early combination antibiotic therapy yields improved survival compared with monotherapy in septic shock: a propensity-matched analysis. Crit Care Med 2010; 38: 1773-1785. https://doi: 10.1097/CCM.0b013e3181eb3ccd.

25. ProCESS Investigators, Yealy DM, Kellum JA, Huang D, Barnato A, Weissfels L, et al. A randomized trial of protocol-based care for early septic shock. N Engl J Med 2014; 370: 1683-1693. https://doi: 10.1056/NEJMoa1401602.

26. PRISM Investigators, Rowan KM, Angus DC, Bailey M, Barnato M, Bellomo R, et al. Early, GoalDirected Therapy for Septic Shock - A Patient-Level Meta-Analysis. N Engl J Med 2017; 376: 22232234. https://doi: 10.1056/NEJMoa1701380.

27. Mouncey PR, Osborn TM, Power GS, Harrison D, Sadique M, Grieve R, et al. Trial of early, goal-directed resuscitation for septic shock. N Engl J Med 2015; 372: 1301-1311. https://doi: 10.1056/NEJMoa1500896.

28. ARISE Investigators, ANZICS Clinical Trials Group, Peake SL, Delaney A, Bailey M, Bellomo R, et al. Goal-directed resuscitation for patients with early septic shock. N Engl J Med 2014; 371: 1496-1506. https://doi: 10.1056/NEJMoa1404380.

29. Levy MM, Fink MP, Marshall JC, Abraham E, Angus D, Cook D, et al. 2001 SCCM/ESICM/ACCP/ATS/SIS International Sepsis Definitions Conference. Crit Care Med 2003; 31: 1250-1256. https://doi: 10.1097/01.CCM.0000050454.01978.3B.

30. Howell MD, Talmor D, Schuetz P, Hunziker S, Jones A, Shapiro N. Proof of principle: the predisposition, infection, response, organ failure sepsis staging system. Crit Care Med 2011; 39: 322327. https://doi: 10.1097/CCM.0b013e3182037a8e.

31. Gai L, Tong Y, Yan B-Q. Research on the diagnostic effect of PCT level in serum on patients with sepsis due to different pathogenic causes. Eur Rev Med Pharmacol Sci 2018; 22: 4238-4242. https://doi: 10.26355/eurrev_201807_15418.

32. Aduen J, Bernstein WK, Khastgir T, Miller J, Kerzner R, Bhatiani A. The use and clinical importance of a substrate-specific electrode for rapid determination of blood lactate concentrations. JAMA 1994; 272: $1678-1685$.

33. Tomar B, Anders H-J, Desai J, Mulay S. Neutrophils and Neutrophil Extracellular Traps Drive Necroinflammation in COVID-19. Cells 2020; 9: E1383. https://doi: 10.3390/cells9061383.

34. Huang Z, Fu Z, Huang W, Huang K. Prognostic value of neutrophil-to-lymphocyte ratio in sepsis: A meta-analysis. Am J Emerg Med 2020; 38: 641-647. https://doi: 10.1016/j.ajem.2019.10.023.

35. Moreno RP, Metnitz B, Adler L, Hoechtl A, Bauer P, Hetnitz P, et al. Sepsis mortality prediction based on predisposition, infection and response. Intensive Care Med 2008; 34: 496-504. https://doi: 10.1007/s00134-007-0943-1.

36. Padkin A, Goldfrad C, Brady AR, Young D, Black N囚RowKan K. Epidemiology of severe sepsis occurring in the first $24 \mathrm{hrs}$ in intensive care units in England, Wales, and Northern Ireland. Crit Care Med 2003; 31: 2332-2338. https://doi: 10.1097/01.CCM.0000085141.75513.2B. 


\section{Tables}

Table 1

Criteria of the Howell-PIRO system

\begin{tabular}{|c|c|c|c|c|c|}
\hline Variable & 0 & 1 & 2 & 3 & 4 \\
\hline \multicolumn{6}{|l|}{ Predisposition } \\
\hline Age (years) & $<65$ & 65 to 80 & $>80$ & & \\
\hline COPD & & Yes & & & \\
\hline Liver disease & & & Yes & & \\
\hline Nursing home resident & & & Yes & & \\
\hline Malignancy & & $\begin{array}{l}\text { Without } \\
\text { metastases }\end{array}$ & $\begin{array}{l}\text { With } \\
\text { metastases }\end{array}$ & & \\
\hline \multicolumn{6}{|l|}{ Infection } \\
\hline \multicolumn{6}{|l|}{ Skin/soft tissue infection } \\
\hline Any other infection & \multirow{2}{*}{\multicolumn{5}{|c|}{ Yes }} \\
\hline Pneumonia & & & & & \\
\hline \multicolumn{6}{|l|}{ Response } \\
\hline Respiratory rate (bpm) & & & \multicolumn{3}{|c|}{$>20$} \\
\hline Bands & & $>5 \%$ & & & \\
\hline Heart rate (bpm) & & & $>120$ & & \\
\hline \multicolumn{6}{|l|}{ Organ dysfunction } \\
\hline $\mathrm{SBP}(\mathrm{mmHg})$ & $>90$ & & 70 to 90 & & \\
\hline BUN (mmol/l) & & & $>7.1$ & & \\
\hline $\begin{array}{l}\text { Respiratory failure/ } \\
\text { hypoxemia }\end{array}$ & & & \multicolumn{3}{|c|}{ Yes } \\
\hline Lactate (mmol/l) & & & \multicolumn{3}{|c|}{$>4.0$} \\
\hline Platelet count $\left(\times 10^{9} / \mathrm{I}\right)$ & & & $<150$ & & \\
\hline
\end{tabular}


Abbreviations: COPD, chronic obstructive pulmonary disease; SBP, systolic blood pressure; BUN, blood urea nitrogen.

\section{Table 2 is available in the Supplementary Files section}

\section{Table 3}

Binary Logistic regression analysis of clinical outcome for septic patients with positive blood culture. 


\begin{tabular}{|c|c|c|c|c|c|c|}
\hline Variables & Predictor & $\beta$ & $S E$ & Wald & $P$ & OR $\square 95 \% \mathrm{Cl}$ \\
\hline \multirow[t]{10}{*}{ ICU-admission } & WBC & 0.176 & 1.453 & 5.632 & 0.018 & 1.192ه1.031-1.379ه \\
\hline & РCT & 0.241 & 0.105 & 5.315 & 0.021 & $1.273 \rrbracket 1.037-1.563 \rrbracket$ \\
\hline & Lac & 1.261 & 0.325 & 15.055 & 0.000 & $3.530 \otimes 1.867-6.676 \rrbracket$ \\
\hline & ALB & -0.142 & 0.046 & 9.501 & 0.002 & $0.867 \rrbracket 0.792-0.949 \rrbracket$ \\
\hline & NLR & 0.077 & 0.034 & 5.205 & 0.023 & $1.080 \otimes 1.011-1.155 \rrbracket$ \\
\hline & SOFA & 0.220 & 0.142 & 2.414 & 0.120 & 2.701ه1.548-4.713区 \\
\hline & MEDS & 0.895 & 0.215 & 17.369 & 0.000 & $2.447 \rrbracket 1.607-3.728 \rrbracket$ \\
\hline & APACHE II & 0.623 & 0.144 & 18.644 & 0.000 & 1.864囚1.405-2.474】 \\
\hline & PIRO & 0.328 & 0.110 & 8.840 & 0.003 & 1.388ه1.118-1.723区 \\
\hline & Constant & -13.820 & 2.939 & 22.112 & 0.000 & \\
\hline \multirow[t]{10}{*}{ MODS } & WBC & 0.362 & 0.129 & 7.861 & 0.005 & 1.436ه1.115-1.849ه \\
\hline & РCT & 0.136 & 0.094 & 2.093 & 0.048 & 1.145冈0.953-1.377ه \\
\hline & Lac & 0.177 & 0.299 & 0.351 & 0.554 & $1.194 \otimes 0.664-2.145 \rrbracket$ \\
\hline & ALB & -0.665 & 0.192 & 11.967 & 0.001 & $0.514 \otimes 0.353-0.750 \rrbracket$ \\
\hline & NLR & 0.270 & 0.093 & 8.452 & 0.004 & $1.310 \otimes 1.092-1.572 \rrbracket$ \\
\hline & SOFA & 0.001 & 0.212 & 0.000 & 0.995 & $1.001 \otimes 0.660-1.518 \rrbracket$ \\
\hline & MEDS & 0.383 & 0.159 & 5.808 & 0.016 & $1.466 \rrbracket 1.074-2.001 \rrbracket$ \\
\hline & APACHE II & 0.372 & 0.162 & 5.286 & 0.022 & $1.450 \otimes 1.056-1.990 \rrbracket$ \\
\hline & PIRO & 0.196 & 0.084 & 5.461 & 0.019 & $1.216 \rrbracket 1.032-1.434 \rrbracket$ \\
\hline & Constant & -18.945 & 4.468 & 19.978 & 0.000 & \\
\hline \multirow[t]{8}{*}{ 28-day-mortality } & WBC & 0.996 & 0.354 & 7.919 & 0.005 & 2.707ه1.353-5.417区 \\
\hline & РCT & 0.306 & 0.098 & 9.764 & 0.002 & $1.358 \rrbracket 1.121-1.644 \rrbracket$ \\
\hline & Lac & 1.822 & 0.701 & 6.753 & 0.009 & $6.184 ه 1.565-24.436 \rrbracket$ \\
\hline & ALB & -0.226 & 0.116 & 3.807 & 0.051 & $0.798 \bowtie 0.636-1.001 \rrbracket$ \\
\hline & NLR & 0.128 & 0.153 & 0.701 & 0.402 & $1.136 \otimes 0.843-1.533 \rrbracket$ \\
\hline & SOFA & 0.092 & 0.157 & 0.340 & 0.560 & $1.096 \rrbracket 0.805-1.492 \rrbracket$ \\
\hline & MEDS & 0.440 & 0.147 & 8.922 & 0.003 & $1.553 \rrbracket 1.163-2.074 \rrbracket$ \\
\hline & APACHE II & 0.484 & 0.163 & 8.832 & 0.003 & $1.623 \rrbracket 1.179-2.234 \rrbracket$ \\
\hline
\end{tabular}




\begin{tabular}{|llllll|} 
PIRO & 0.322 & 0.123 & 6.884 & 0.009 & $1.380 \rrbracket 1.085-1.754 \rrbracket$ \\
Constant & -16.492 & 3.630 & 20.646 & 0.000 & \\
\hline
\end{tabular}

$\mathrm{Cl}$, confidence interval; OR, odds ratio; SE, standard error. WBC, white blood cell; PCT, procalcitonin; Lac, lactate; ALB, albumin; NLR, neutrophils and lymphocytes ratio; SOFA, Sequential Organ Failure Assessment; APACHE II, Acute Physiology and Chronic Health Evaluation Il; MEDS, Mortality in emergency department sepsis; PIRO, Predisposition, Infection, Response, and Organ dysfunction

\section{Table 4}

Statistical data of ROC curve in predicting ICU-admission in patients with positive blood culture.

\begin{tabular}{|c|c|c|c|c|c|c|}
\hline Variables & AUC $(95 \% \mathrm{Cl})$ & $S E$ & $P$ & Cut off & Sensitivity & Specificity \\
\hline WBC & $0.725 \rrbracket 0.496-0.954 \rrbracket$ & 0.117 & 0.075 & 12.75 & 0.800 & 0.750 \\
\hline РCT & $0.620 \rrbracket 0.533-0.708 \rrbracket$ & 0.045 & 0.009 & 6 & 0.294 & 0.977 \\
\hline Lac & $0.637 \rrbracket 0.454-0.820 \rrbracket$ & 0.093 & 0.125 & 2.25 & 0.500 & 0.913 \\
\hline ALB & $0.759 \rrbracket 0.574-0.945 \rrbracket$ & 0.095 & 0.015 & 26.35 & 0.875 & 0.636 \\
\hline NLR & $0.615 \varangle 0.449-0.782 \rrbracket$ & 0.085 & 0.225 & 5.03 & 0.950 & 0.353 \\
\hline MEDS & $0.740 \rrbracket 0.663-0.817 \rrbracket$ & 0.039 & 0.000 & 8.5 & 0.646 & 0.759 \\
\hline APACHE II & $0.780 \rrbracket 0.709-0.850 \rrbracket$ & 0.036 & 0.000 & 15.5 & 0.468 & 0.975 \\
\hline PIRO & $0.751 \rrbracket 0.674-0.828 \rrbracket$ & 0.039 & 0.000 & 11.5 & 0.684 & 0.759 \\
\hline PCT+MEDS & $0.772 \varangle 0.698-0.846 \rrbracket$ & 0.038 & 0.000 & 0.39 & 0.924 & 0.557 \\
\hline PCT+APACHE II & $0.821 \rrbracket 0.757-0.885 \rrbracket$ & 0.033 & 0.000 & 0.37 & 0.899 & 0.608 \\
\hline PCT+PIRO & $0.795 \rrbracket 0.723-0.867 \rrbracket$ & 0.037 & 0.000 & 0.45 & 0.911 & 0.633 \\
\hline
\end{tabular}

Abbreviations: WBC, white blood cell; PCT, procalcitonin; Lac, lactate; ALB, albumin; NLR, neutrophils and lymphocytes ratio; APACHE II, Acute Physiology and Chronic Health Evaluation II; MEDS, Mortality in emergency department sepsis; PIRO, Predisposition, Infection, Response, and Organ dysfunction

Table 5

Statistical data of ROC curve in predicting MODS in patients with positive blood culture. 


\begin{tabular}{|c|c|c|c|c|c|c|}
\hline Variables & AUC $(95 \% \mathrm{Cl})$ & $S E$ & $P$ & Cut off & Sensitivity & Specificity \\
\hline WBC & $0.879 \rrbracket 0.819-0.938 \rrbracket$ & 0.030 & 0.000 & 12.75 & 0.911 & 0.878 \\
\hline РСТ & $0.664 \rrbracket 0.584-0.745 \rrbracket$ & 0.041 & 0.000 & 1.01 & 0.911 & 0.426 \\
\hline ALB & $0.789 \rrbracket 0.722-0.856 \rrbracket$ & 0.034 & 0.000 & 28.10 & 0.851 & 0.620 \\
\hline NLR & $0.727 \rrbracket 0.653-0.801 \rrbracket$ & 0.038 & 0.000 & 7.32 & 0.873 & 0.511 \\
\hline MEDS & $0.701 \rrbracket 0.622-0.779 \rrbracket$ & 0.040 & 0.000 & 12.5 & 0.43 & 0.957 \\
\hline APACHE II & $0.761 \rrbracket 0.691-0.832 \rrbracket$ & 0.036 & 0.000 & 12.5 & 0.658 & 0.723 \\
\hline PIRO & $0.811 \rrbracket 0.746-0.876 \rrbracket$ & 0.033 & 0.000 & 12.5 & 0.544 & 0.957 \\
\hline PCT+MEDS & $0.758 \rrbracket 0.685-0.831 \rrbracket$ & 0.037 & 0.000 & 0.53 & 0.582 & 0.862 \\
\hline PCT+APACHE II & $0.794 \bowtie 0.727-0.862 \rrbracket$ & 0.034 & 0.000 & 0.47 & 0.684 & 0.787 \\
\hline PCT+PIRO & $0.837 \rrbracket 0.776-0.898 \rrbracket$ & 0.031 & 0.000 & 0.48 & 0.696 & 0.883 \\
\hline
\end{tabular}

Abbreviations: WBC, white blood cell; PCT, procalcitonin; Lac, lactate; ALB, albumin; NLR, neutrophils and lymphocytes ratio; APACHE II, Acute Physiology and Chronic Health Evaluation II; MEDS, Mortality in emergency department sepsis; PIRO, Predisposition, Infection, Response, and Organ dysfunction

Table 6

Statistical data of ROC curve in predicting 28-day-mortality in patients with positive blood culture.

\begin{tabular}{|c|c|c|c|c|c|c|}
\hline Variables & AUC $(95 \% \mathrm{Cl})$ & $S E$ & $P$ & Cut off & Sensitivity & Specificity \\
\hline WBC & $0.789 \llbracket 0.673-0.905 \rrbracket$ & 0.059 & 0.000 & 14.05 & 0.767 & 0.896 \\
\hline РCT & $0.782 \rrbracket 0.683-0.882 \rrbracket$ & 0.051 & 0.000 & 1.05 & 0.933 & 0.521 \\
\hline Lac & $0.699 \llbracket 0.561-0.838 \rrbracket$ & 0.071 & 0.003 & 2.25 & 0.567 & 0.937 \\
\hline MEDS & $0.745 \rrbracket 0.627-0.863 \rrbracket$ & 0.060 & 0.000 & 12.5 & 0.400 & 0.979 \\
\hline APACHE ॥ & $0.805 \rrbracket 0.707-0.904 \rrbracket$ & 0.050 & 0.000 & 15.5 & 0.633 & 0.875 \\
\hline PIRO & $0.831 \otimes 0.742-0.920 \rrbracket$ & 0.045 & 0.000 & 11.5 & 0.867 & 0.708 \\
\hline PCT+MEDS & 0.812ه0.705-0.919ه & 0.055 & 0.000 & 0.50 & 0.667 & 0.937 \\
\hline PCT+APACHE II & $0.866 \rrbracket 0.789-0.943 \rrbracket$ & 0.039 & 0.000 & 0.23 & 0.933 & 0.687 \\
\hline PCT+PIRO & $0.885 \bowtie 0.810-0.961 \rrbracket$ & 0.039 & 0.000 & 0.39 & 0.833 & 0.896 \\
\hline
\end{tabular}

Abbreviations: WBC, white blood cell; PCT, procalcitonin; Lac, lactate; APACHE II, Acute Physiology and Chronic Health Evaluation II; MEDS, Mortality in emergency department sepsis; PIRO, Predisposition, Infection, Response, and Organ dysfunction 
Figures

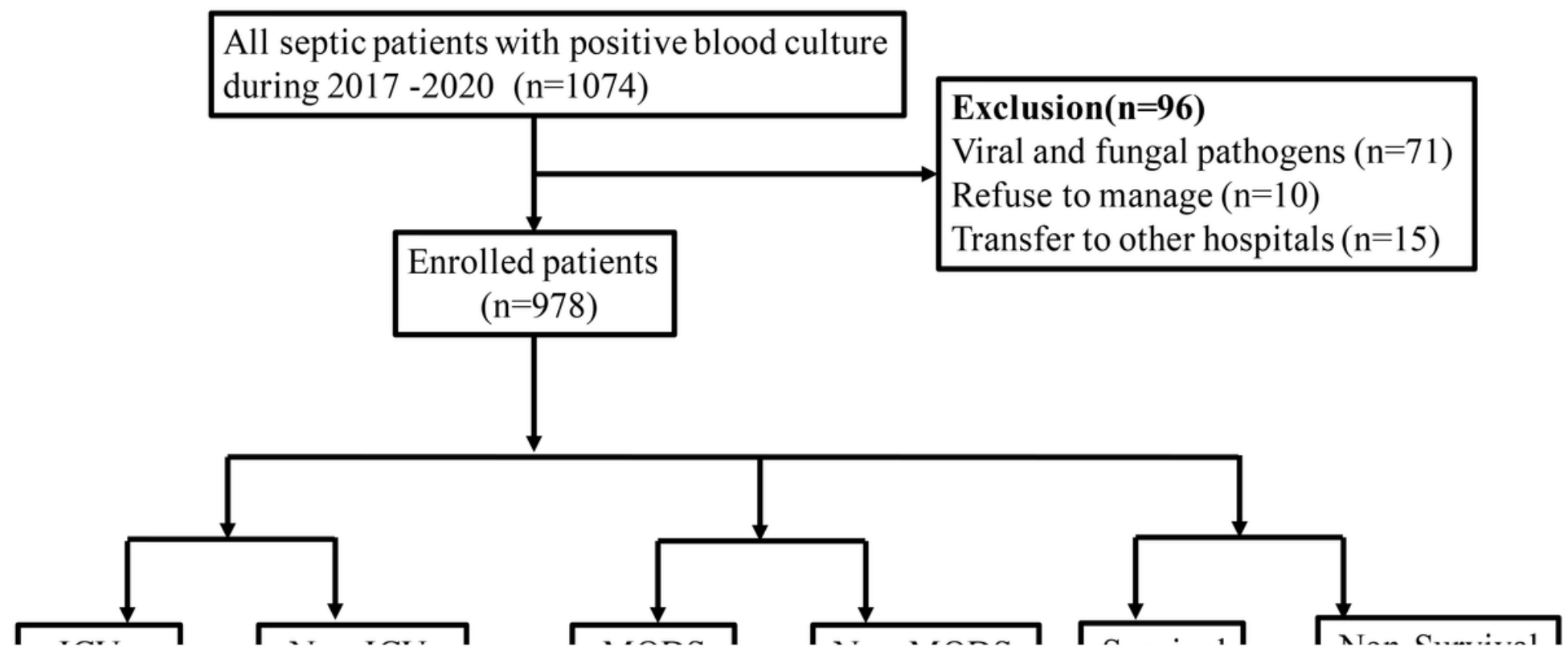

Figure 1

Flowchart of the study population. 
A
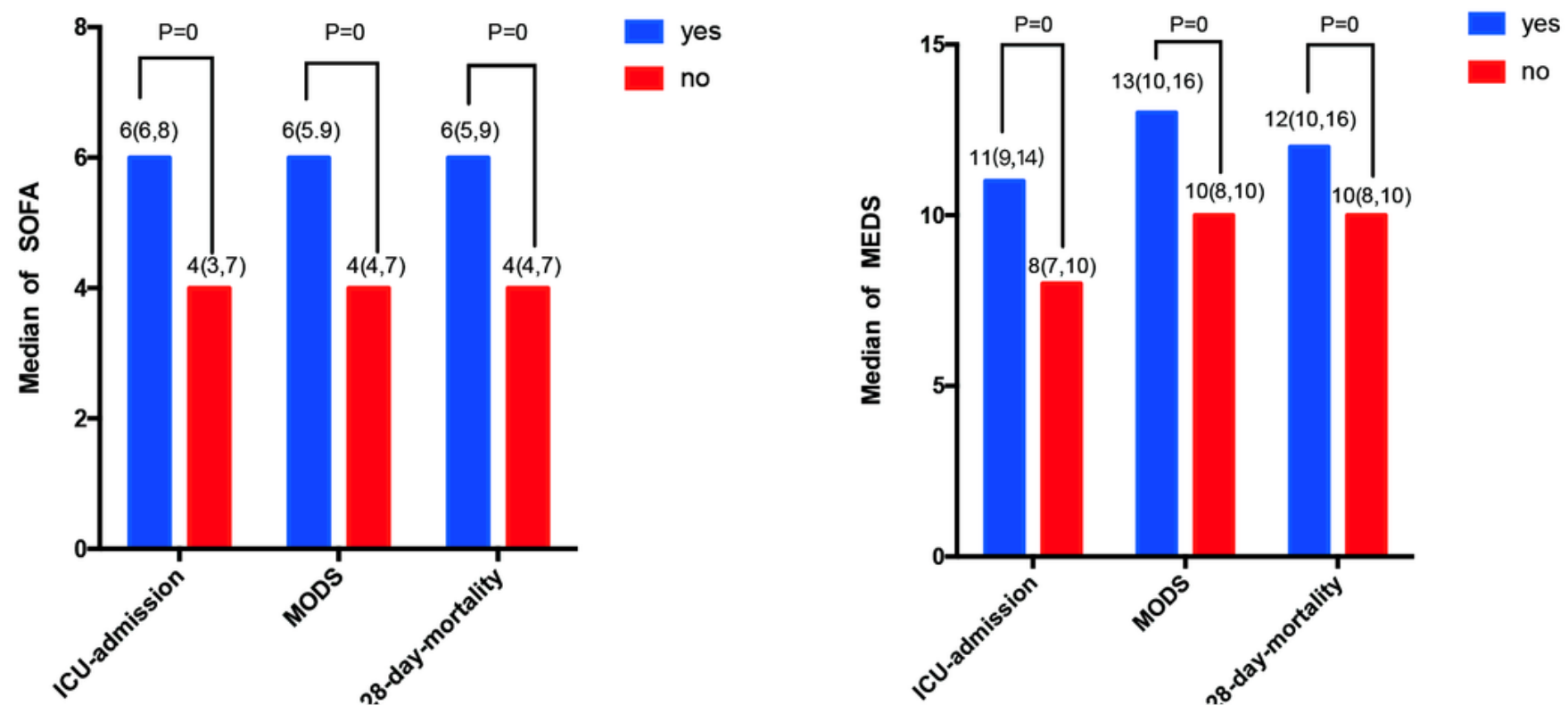

Figure 2

The median levels of SOFA, MEDS and PIRO scores, and mean level of APACHE II score in ICU-admission, developing of MODS and 28-day mortality groups. (A) The median level of SOFA score in ICU-admission, developing of MODS and 28-day mortality groups. (B) The median level of MEDS score in ICU-admission, developing of MODS and 28-day mortality groups. (C)The mean level of APACHE II score in ICU- 
admission, developing of MODS and 28-day mortality groups. (D) The median level of PIRO score in ICUadmission, developing of MODS and 28-day mortality groups.

A

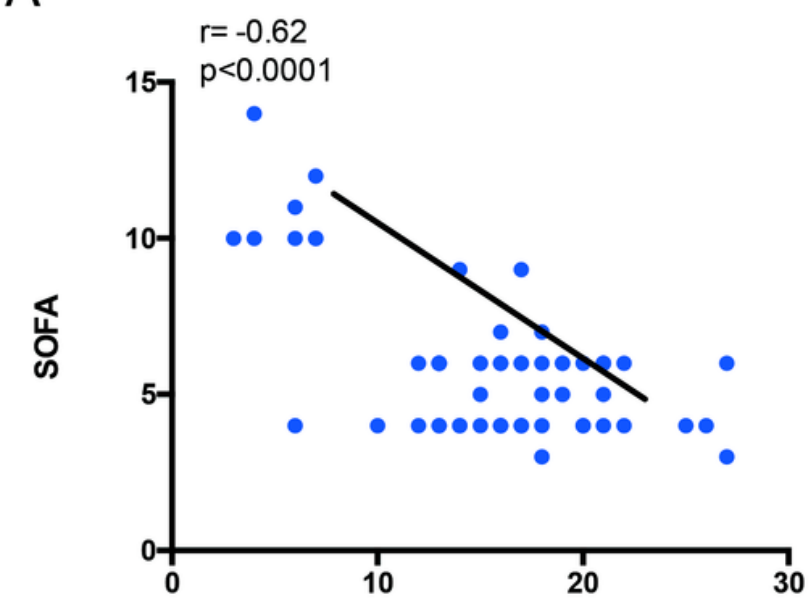

C

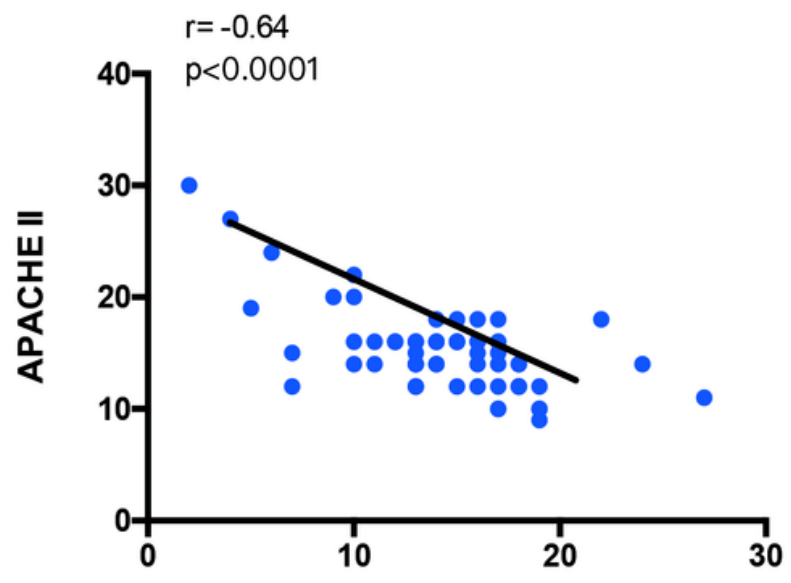

Survival
B



D

Survival

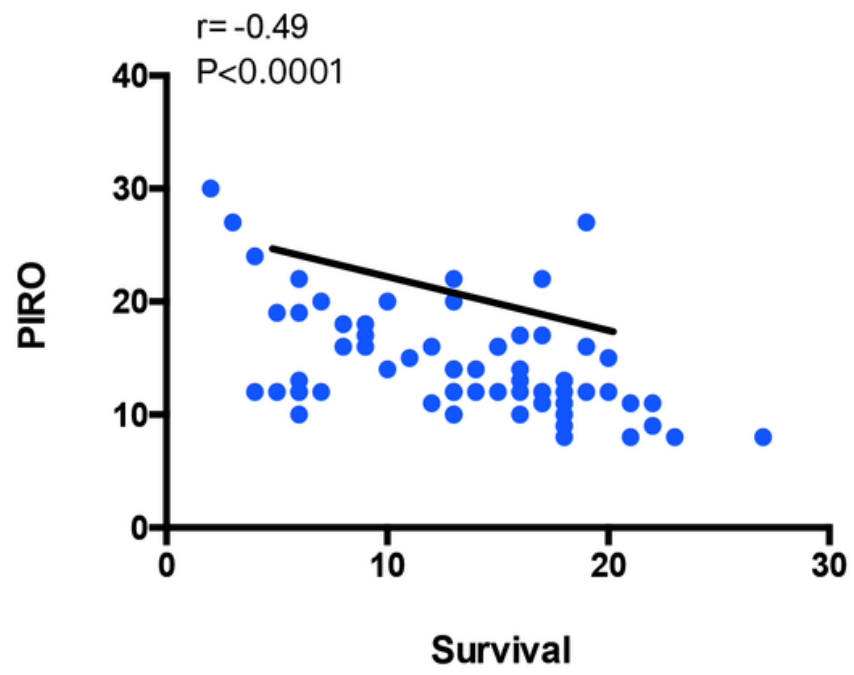

Figure 3

Spearman's correlations between 28-day mortality and SOFA score (A); MEDS score (B); APACHE II score (C); PIRO score (D) in patients with positive blood cultures. 
A

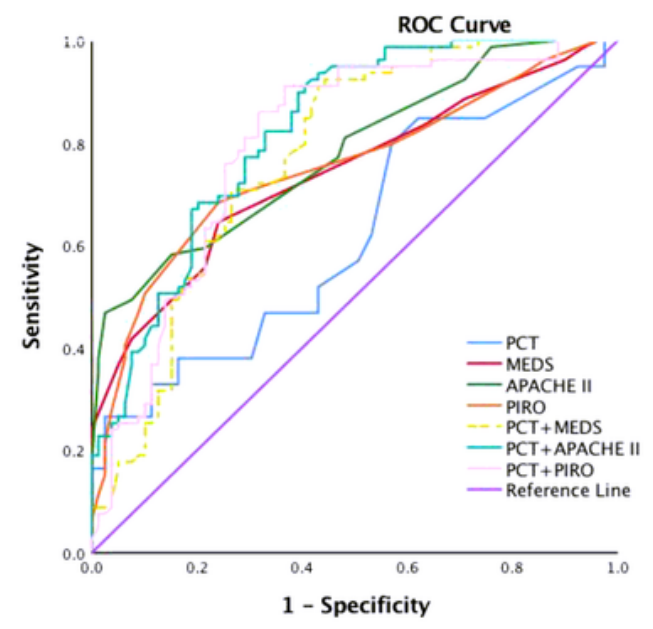

B

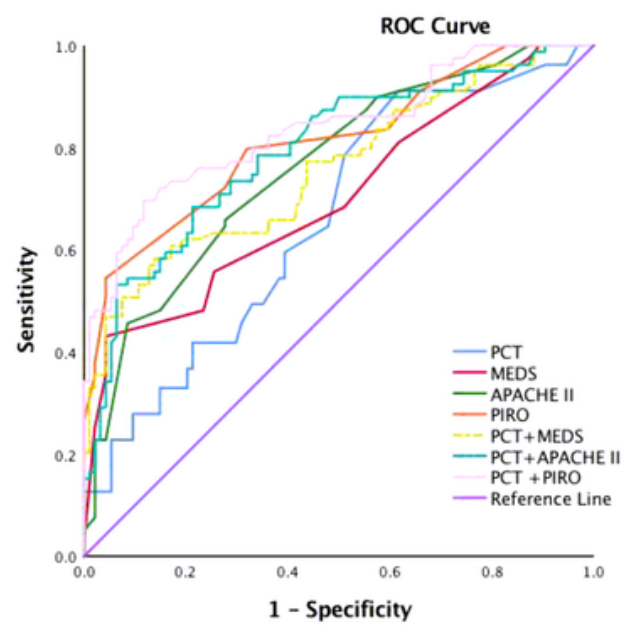

C

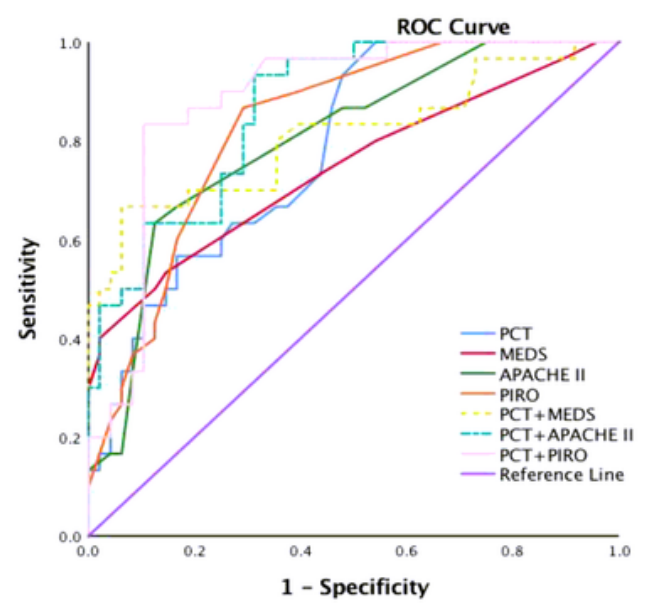

\section{Figure 4}

The ROC curves of PCT combined with severe scores for predicting outcomes in positive blood culture septic patients for ICU-admission, developing of MODS and 28-day mortality. (A) The ROC curves of PCT combined with severe scores in positive blood culture septic patients for ICU-admission; (B) The ROC curves of PCT combined with severe scores in positive blood culture septic patients for developing of 
MODS; (C) The ROC curves of PCT combined with severe scores in positive blood culture septic patients for 28-day mortality.

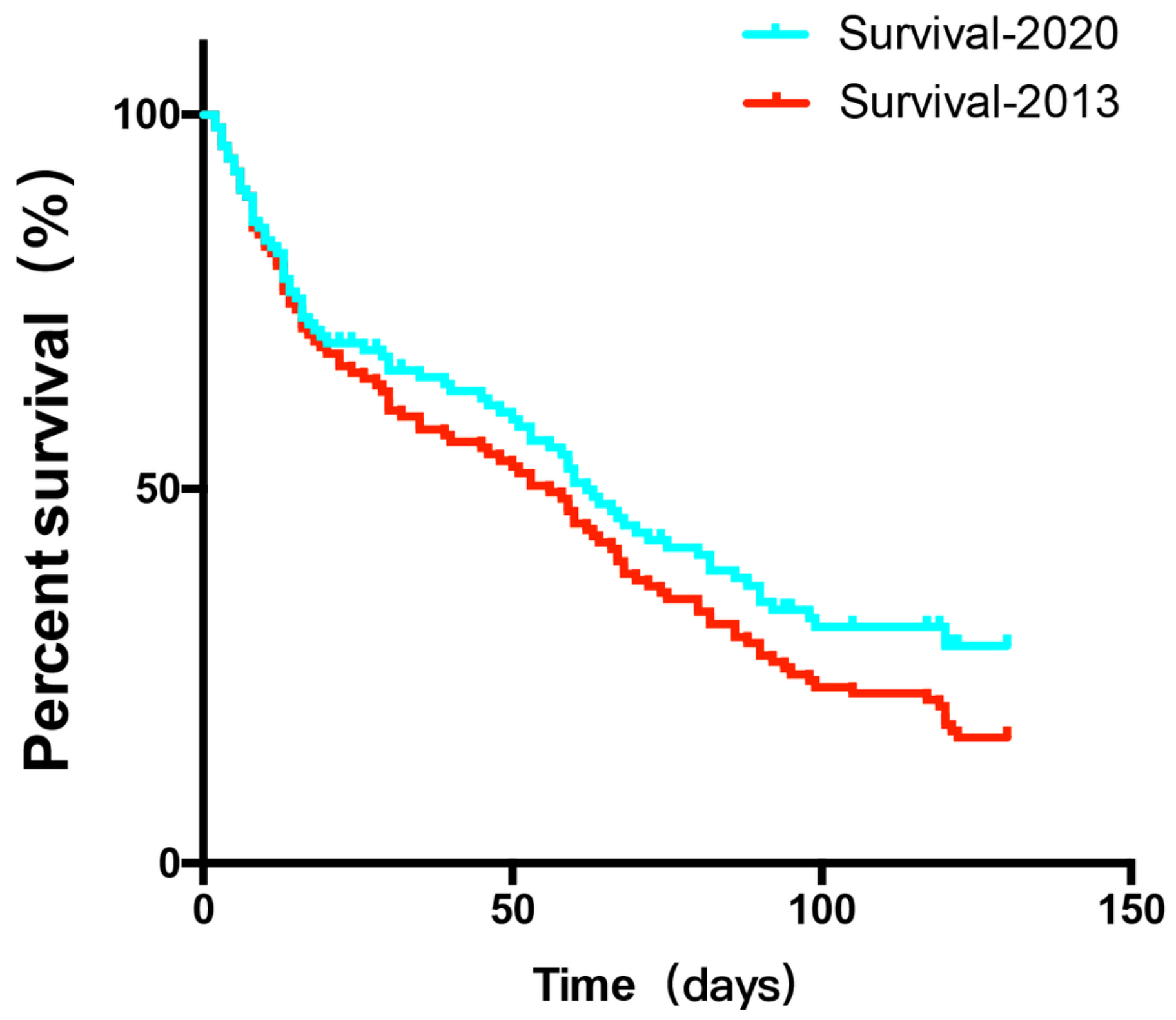

Figure 5

The comparison of survival time between 2013 and 2020.

\section{Supplementary Files}

This is a list of supplementary files associated with this preprint. Click to download.

- Table2.docx 